\title{
Amino Acid Neurotransmission between Fimbria-Fornix Fibers and Neurons in the Lateral Septum of the Rat: A Microiontophoretic Study
}

\author{
M. JoËLS AND I. J. A. URBAN ${ }^{1}$ \\ Rudolf Magnus Institute for Pharmacology, University of Utrecht, Vondellaan 6, \\ 3521 GD Utrecht, The Netherlands
}

Received August 3, 1983; revision received October 18, 1983

\begin{abstract}
We investigated the nature of the excitatory amino acid and the type of amino acid receptor involved in the projection of fimbria-fornix ( $\mathrm{f}-\mathrm{fx}$ ) fibers on neurons in the lateral septal complex (LSC) of the rat. It appeared that neurons which were strongly orthodromically activated (SOA) by stimulation of $\mathrm{fi}-\mathrm{fx}$ fibers were excited by glutamate (GLU) and aspartate (ASP) at much lower ejecting currents than neurons which were only weakly orthodromically excited. In addition, GLU was a stronger agent than ASP, particularly in SOA septal cells. Two amino acid antagonists tested, glutamic acid diethylester (GDEE) and 2-amino-5-phosphonovaleric acid (2-APV), selectively antagonized responses to the amino acid agonists quisqualate (QUIS) and $N$-methylD-aspartate (NMDA), respectively. They also depressed GLU- and ASP-induced responses, although in that case the antagonists frequently had to be expelled with currents higher than those needed to block QUIS- and NMDA-evoked excitations. Furthermore, GDEE frequently antagonized GLU-induced responses better than ASPevoked excitations, whereas 2-APV often blocked responses to ASP more effectively than those to GLU. It was observed that GDEE, ejected with currents that blocked responses to QUIS reversibly, decreased the number of synaptic responses induced in SOA cells by fi-fx stimuli. Synaptically induced excitation in these neurons was consistently unaffected by $2-\mathrm{APV}$, even when the antagonist was expelled with high currents. According to these results, LSC neurons, in particular the SOA neurons, are more
\end{abstract}

\footnotetext{
Abbreviations: $\mathrm{fi}-\mathrm{fx}$-fimbria-fornix; LSC-lateral septal complex; SOA, WOA-strongly, weakly orthodromically activated; GLU-glutamate; ASP_aspartate; GDEE-glutamic acid diethylester; 2-APV-2-amino-5-phosphonovaleric acid; QUIS-quisqualate; NMDA $-N$-methylD-aspartate; KA-kainic acid.

${ }^{1}$ The investigations were supported by the Foundation for Medical Research FUNGO which is subsidized by the Netherlands Organization for Advancement of Pure Research (Z.W.O., grant 13-31-045, to I. J. A. Urban).
} 
readily activated by GLU than by ASP. Monosynaptic excitations elicited in SOA septal cells by fi-fx stimuli appear to be predominantly if not exclusively mediated by QUIS receptors. There are indications that GLU-induced responses in the LSC neurons are presumably mediated by the QUIS receptors. From these data it may be inferred that GLU rather than ASP is the transmitter involved in the projection of $f-f x$ fibers on LSC neurons.

\section{INTRODUCTION}

Electrophysiological investigations have revealed that much of the innervation which the lateral septal complex (LSC) receives from the hippocampal formation by way of the fimbria-fornix (fi-fx) fibers, is excitatory $(9,10,20$, 23). Data indicating that the innervation is mediated by an excitatory amino acid were obtained from biochemical studies. Electrical stimuli delivered to $\mathrm{fi}-\mathrm{fx}$ fibers preincubated with $\left[{ }^{3} \mathrm{H}\right]$ aspartate induced a $\mathrm{Ca}^{2+}$-dependent release of the amino acid marker from fi-fx terminals (18). In addition, extirpation of the entire hippocampus, destruction of the hippocampal pyramids with kainic acid, or transection of the fi-fx pathway markedly reduced the amount of endogenous glutamate (GLU) and the high-affinity uptake of this amino acid in the dorsolateral septum $(13,25,30,32,34)$. In that experimental design, however, the uptake mechanism of GLU does not distinguish between GLU- and aspartate (ASP)-using fibers (2).

Recently, we examined electrophysiologically the excitatory fi-fx projection in more detail (16). It appeared that neurons situated in the dorsal part of the $\mathrm{LSC}$ receive a considerably stronger excitatory input along $\mathrm{fi}-\mathrm{fx}$ fibers than do neurons in the ventral part of the LSC. The dorsally located cells which were strongly orthodromically activated (SOA) by electrical stimulation of the fi-fx fibers, were found to be more responsive to iontophoretically administered GLU than neurons which were only weakly orthodromically activated (WOA). Glutamic acid diethylester (GDEE), an excitatory amino acid antagonist (22), suppressed both the GLU- and synaptically elicited excitations in LSC neurons. These results suggested that amino acid receptors are present on LSC cells, that the receptors are presumably involved in the GLU- and synaptically induced excitations and they thus support the hypothesis that fi-fx fibers innervating LSC neurons use an amino acid as transmitter. On the basis of these biochemical and electrophysiological experiments, however, it can not be decided which of the two putative excitatory amino acid transmitters, GLU or ASP, may actually be involved in the fifx projection to the LSC.

Two electrophysiological approaches can be used to further investigate which of the excitatory amino acids, GLU and ASP, and what type of amino acid receptor mediates the excitations induced in LSC cells by fi-fx stimulation. First, it can be investigated whether or not a difference in sensitivity to 
iontophoretically administered GLU and ASP exists between neurons receiving a strongly excitatory input along the $\mathrm{fi}-\mathrm{fx}$ fibers, i.e., the SOA neurons, and neurons which do not receive this strong input. A difference in sensitivity may be expected if one of the amino acids acts as transmitter in this pathway. The second approach is executed with the aid of recently developed specific amino acid agonists and antagonists. Two types of excitatory amino acid receptors have been clearly defined using these compounds: one selectively activated by quisqualate (QUIS) and preferentially blocked by GDEE, the other activated by $N$-methyl-D-aspartate (NMDA) and antagonized by 2 amino-5-phosphonovaleric acid (2-APV) [see for review (20,33)]. The existence of a third type of receptor activated by kainic acid (KA) was inferred from observations showing that neuronal excitations induced by KA could not be antagonized with either of the antagonists $(8,15,22)$. The natural amino acids, GLU and ASP, can activate all types of receptors, but there is evidence that GLU acts more effectively on the QUIS type of receptors whereas ASP exhibits a stronger action upon NMDA receptors (5). Determination of the type of receptor mediating the transmission between $\mathrm{fi}-\mathrm{fx}$ fibers and LSC cells may therefore provide some information about the amino acid actually involved in this projection.

We used both methods to study the nature of the excitatory amino acid and the type of amino acid receptor involved in neurotransmission between $\mathrm{fi}-\mathrm{fx}$ terminals and LSC neurons. Thus, we examined the responsiveness of SOA and WOA septal cells to iontophoretically applied GLU and ASP. Subsequently, we investigated the type of amino acid receptor involved in the fi-fx projection, using the amino acid agonists, QUIS and NMDA, and the antagonists, GDEE and 2-APV.

\section{MATERIALS AND METHODS}

The experiments were carried out on 23 male Wistar rats. The animals $(250$ to $300 \mathrm{~g})$ were anesthetized with urethane $(1.5 \mathrm{~g} / \mathrm{kg})$, provided with a tracheal cannula, and placed in a stereotaxic instrument. After craniotomy, the neocortex and corpus callosum overlying the septum and dorsal hippocampus were gently removed by suction; local bleeding was stopped with hemostatic sponges. The exposed surface of the septum was covered with agar $\left(37^{\circ} \mathrm{C}\right)$. In this preparation, the intersection of the anterior border of the hippocampal commissure with the midline served as reference zero for the visually guided placement of the recording electrodes. Body temperature was maintained at about $36^{\circ} \mathrm{C}$. At least $3 \mathrm{~h}$ elapsed between surgery and the experiment.

Stimulation and Recording. Four pairs of electrodes (stainless-steel wire $100 \mu \mathrm{m}$ in diameter, coated with Trimel except for the tip) were unilaterally 
inserted into the fi-fx immediately behind the caudal border of the hippocampal commissure. The length of the stimulation electrodes was adjusted to the profile of the $f-f x$ so that both the dorsal and the ventral aspects of the $\mathrm{fi}-\mathrm{fx}$ could be stimulated during the experiments. These electrodes served for the synaptical activation of single units in the LSC.

Action potentials of single neurons were recorded with glass micropipets (filamented Clark glass, $1 \mathrm{~mm}$ diameter) filled with $3 \mathrm{M} \mathrm{NaCl}$. The recording micropipets were glued to 7 -barrel multipipets so that their tips protruded in front of the multipipet by 30 to $50 \mu \mathrm{m}$. The signal from the recording electrodes was filtered (low- and high-frequency cut-offs at 200 and $5000 \mathrm{~Hz}$, respectively) and fed into registering instruments consisting of an oscilloscope, audio-amplifier, magnetic tape, and window discriminator. Single and integrated pulses of the window discriminator were recorded on polygraphic paper and used for quantification of the single-unit data.

Drugs. The channels of the multibarrel pipets were filled with solutions of the following drugs: L-aspartic acid (ASP, Sigma, $0.25 \mathrm{M}$ adjusted to $p \mathrm{H}$ 8 with $\mathrm{NaOH}$, expelled as anion), L-glutamic acid (GLU, Sigma, $0.25 M$ adjusted to $p \mathrm{H} 8$ with $\mathrm{NaOH}$, anion), L-glutamic acid diethylester (GDEE, Fluka, $0.5 \mathrm{M}, p \mathrm{H} \mathrm{4}$, cation), $\mathrm{MgCl}_{2}$ (Merck, $0.5 \mathrm{M}, \mathrm{pH}$ unadjusted, cation), all aquous solutions; quisqualic acid (QUIS, Sigma, $5 \mathrm{mM}$ ), $N$-methyl-Daspartate (NMDA, CRB, $50 \mathrm{mM}$ ) and \pm 2 -amino-5-phosphonovaleric acid (2-APV, CRB, $50 \mathrm{mM}$ ) all dissolved in $165 \mathrm{mM} \mathrm{NaCl}$, adjusted to $p \mathrm{H} 7$ with $\mathrm{NaOH}$, and ejected as anion. Automatic current balancing was through a barrel containing $3 \mathrm{M} \mathrm{NaCl}$. Impedances of the drug channels ranged from 10 to $40 \mathrm{M} \Omega$. Retaining currents were about $10 \mathrm{nA}$.

Experimental Protocol and Data Analysis. Neurons were sampled in penetrations made through the LSC at $1500 \mu \mathrm{m}$ anterior to the reference zero and 500 to $700 \mu \mathrm{m}$ lateral of the midline. To exclude artefacts due to mechanical stimulation, single units were approached with extreme care. As soon as single-unit activity was discerned, the electrode assembly was maintained in place for about $10 \mathrm{~min}$. Next the electrode was slowly lowered until the signal to noise ratio became at least two, the spontaneous activity remaining unaltered on approaching the neuron.

Prior to the iontophoretic application of drugs, all neurons were subjected to a test procedure to establish the input-output relationship of the neurons to fi-fx stimulation. The LSC neurons which displayed an excitatory response to stimulation of $\mathrm{f}-\mathrm{fx}$ fibers with a latency of about $5 \mathrm{~ms}$, were classified as strongly orthodromically activated (SOA) cells if (i) the cells were excited by low-intensity stimuli (usually less than $100 \mu \mathrm{A}$ ) delivered to various pairs of the stimulating electrodes, (ii) the responses appeared at a stable latency, and (iii) the responses followed stimulus frequencies greater than $50 \mathrm{~Hz}$ with a stimulus-response ratio equal to one. Other neurons responded to stimulation 
at comparable latencies, but in that case the latency of the responses to repeated stimuli lacked stability, not every stimulus was followed by a response, the stimulus intensity for eliciting an excitatory response was much higher $(500 \mu \mathrm{A})$, and a further increase of the stimulus intensity did not improve the stimulus-response ratio of those neurons. These cells were classified as weakly orthodromically activated (WOA) neurons. The effect of GLU, ASP, QUIS, and NMDA on the spontaneous activity of these neurons was studied in a test procedure in which the compounds were administered alternatedly during 10-s ejection pulses at fixed intervals ( 30 or $60 \mathrm{~s}$ ). The action of a compound was considered to be excitatory if spontaneous activity was increased by at least $50 \%$ in two subsequent applications. GLU and ASP were also studied in a test sequence in which responses to the amino acids were examined before, during, and after iontophoretic administration of $\mathrm{Mg}^{2+}$.

The potency of GLU relative to that of ASP was determined from the ratio of equieffective ejection currents. The amino acids were ejected either alternatedly or in two separate runs at fixed 60-s intervals, using currents that produced stable, submaximal responses. Approximately equal numbers of SOA and WOA neurons were sampled with each electrode, thus minimizing the influence from variations in the physical properties of the electrodes $(3$, $11,19)$. The results were analyzed statistically with Student's $t$ test.

The action of 2-APV and GDEE on the amino acid-induced responses was studied in a procedure in which the responses of a neuron to 10-s pulses of the agonists given at 60 -s intervals were examined before, during, and after the iontophoretic release of the antagonist. Antagonism was defined as a reduction by at least $50 \%$ of the response to the agonist during release of the antagonist in at least two subsequent runs. The effect of 2-APV and GDEE on synaptically induced excitations was examined in a test in which 32 stimuli were delivered to the $\mathrm{fi}-\mathrm{fx}$ before, during, and after iontophoretic release of the antagonists.

The threshold stimulus intensity was the intensity needed to induce an orthodromic response in the neuron under study in about $50 \%$ of the cases. The antagonists were first expelled with currents which were sufficient to antagonize excitations induced in the neuron by the appropriate agonist. If the antagonist failed to affect the synaptic response at this current level, the expelling currents were increased and the procedure repeated. Poststimulus histograms of the neuronal response before, during, and after administration of the antagonist were computed in a computer average transient set into the poststimulus histogram mode.

Histology. At termination of each experiment, the recording position of the micropipet was marked by placing a pontamine sky blue spot $(10 \mu \mathrm{A}$ for $15 \mathrm{~min}$ ). The position of the stimulating electrodes was marked by iron deposition and the Prussian blue reaction. The animals were killed with an 
overdose of pentobarbital (Nembutal) and perfused with $10 \%$ Formalin in saline. Placement of the recording and stimulating electrodes was reconstructed on frontal frozen sections $(60 \mu \mathrm{m})$ stained with $0.1 \%$ cresyl violet.

\section{RESULTS}

Action of L-Glutamate and L-Aspartate on Neurons of the Lateral Septal Complex. Iontophoretically administered GLU and ASP induced excitatory responses in almost all neurons tested in the LSC, commencing 3 to $5 \mathrm{~s}$ after the onset of the iontophoretic release and terminating rapidly after the end of the release (see an example in Fig. 1). Iontophoretic administration of $\mathrm{Mg}^{2+}$ depressed or completely abolished spontaneous activity in 19 LSC neurons tested. In all cases tested, however, these cells still responded to GLU $(N=14)$ and ASP $(N=9)$ during the release of $\mathrm{Mg}^{2+}$ ions. When the same or nearly the same number of SOA and WOA cells were tested with each
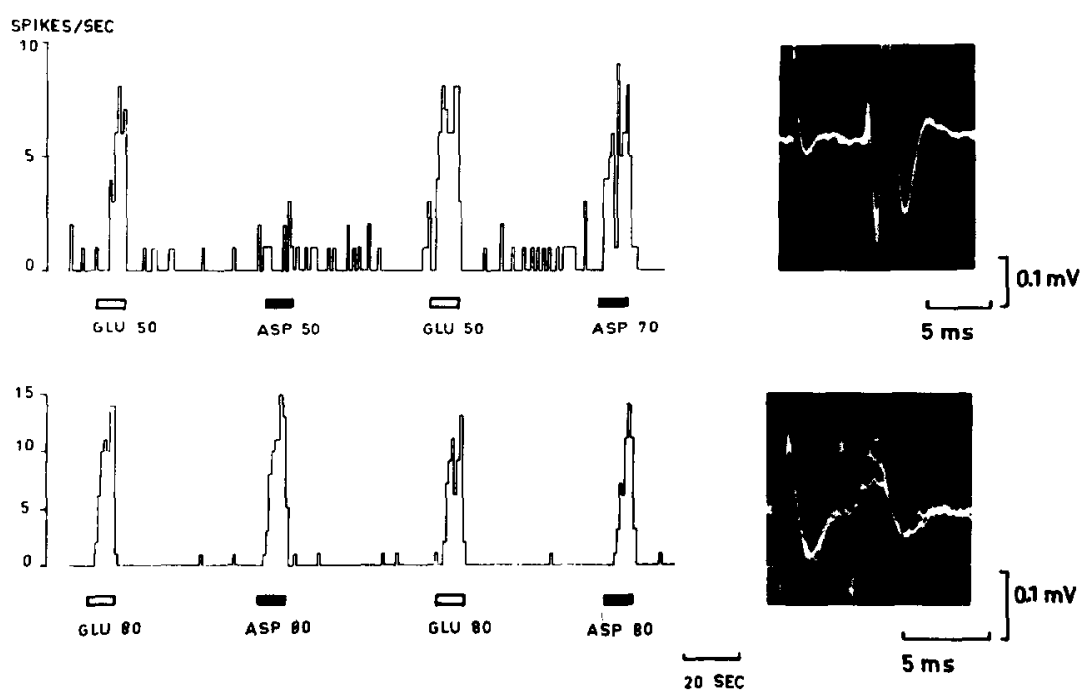

FiG. 1. Responses to L-glutamate (GLU) and L-aspartate (ASP) established in a lateral septal neuron receiving strongly orthodromic activation along fimbria-fornix ( $f$ - $f x$ ) fibers (upper tracing) and a neuron receiving only weakly orthodromic input (lower tracing). The neurons were sampled with the same micropipet assembly during one penetration. The ordinate represents the number of spikes/s. Horizontal bars show the duration of applications, the numbers below the bars representing the ejecting currents (in $\mathrm{nA}$ ) used to obtain approximately equipotent responses for GLU and ASP. Upper inset - three superimposed oscilloscope sweeps of a strongly orthodromically activated neuron. Unit response was followed by a large field potential characteristic for the locus where this neuron was encountered (450 $\mu \mathrm{m}$ below the septal surface). Lower inset-three superimposed oscilloscope sweeps of the weakly activated neuron indicating the response of the cell to fi-fx stimulation. At this depth $(1200 \mu \mathrm{m})$ the field potential was markedly reduced. 
micropipet assembly, it appeared that the mean GLU-ejecting current needed to induce submaximal excitation in SOA cells was markedly less (53\%) than the corresponding current needed for activation of WOA cells. A less pronounced difference $(72 \%)$ in mean expelling currents for those two groups of neurons was observed in the case of ASP (see Table 1). A difference in the action of GLU and ASP on SOA and WOA cells also arose from the mean GLU:ASP current ratios in both groups of neurons. The ratio for SOA cells was significantly smaller $(t=2.76)$ than the ratio for the other group of neurons (see an example in Fig. 1). Interestingly, for eliciting comparable responses with GLU and ASP in SOA cells, ASP had to be expelled on average with a $60 \%$ higher current than GLU (see Table 1), whereas in WOA cells the mean ASP-expelling current was only about $20 \%$ higher than that used for GLU.

Action of Selective Agonists. In 35 of 38 cells tested, both NMDA and QUIS induced excitatory responses, characterized by a slow onset (6 to $8 \mathrm{~s}$ ) and offset (about $5 \mathrm{~s}$; see an example in Fig. 2). In some cases these NMDAand QUIS-induced responses lasted to $30 \mathrm{~s}$ after termination of the iontophoretic release. Of the remaining neurons, two were excited by NMDA but not by QUIS, and one was exclusively activated by QUIS.

Mean NMDA- and QUIS-expelling currents were computed for SOA and WOA neurons collected with electrodes sampling nearly equal numbers of neurons (see Table 1). The SOA cells were activated by QUIS at significantly $(t=2.21)$ lower currents than were WOA cells. With NMDA also, the computed mean current needed to induce excitatory responses in SOA cells was lower than the mean current needed in WOA cells. However, as indicated by the SE, the NMDA-expelling currents varied considerably among the WOA cells.

Effect of Glutamic Acid Diethylester and 2-Amino-5-phosphonovaleric Acid on the Action of Selective Agonists. In 15 of 17 neurons tested, QUIS-induced

\section{TABLE 1}

Mean Expclling Current \pm SE for Quisqualate (QUIS), $N$-Mcthyl-D-aspartatc (NMDA), LGlutamate (GLU), and L-Aspartate (ASP) and the Mean Potency Ratio (GLU-Current:ASPCurrent) Obtained for Two Distinct Populations of Lateral Septal Neurons ${ }^{a}$

\begin{tabular}{llllll}
\hline & GLU current & ASP current & Potency ratio & QUIS current & NMDA current \\
\hline SOA cells & $30.29 \pm 5.59(21)$ & $49.19 \pm 6.91(21)$ & $0.60 \pm 0.06(21)$ & $26.22 \pm 6.21(9)$ & $25.50 \pm 4.55(8)$ \\
WOA cells & $57.32 \pm 7.41(19)^{*}$ & $68.68 \pm 6.55(19)$ & $0.82 \pm 0.05(19)^{*}$ & $48.00 \pm 7.80(7)$ & $50.00 \pm 14.23(6)$ \\
\hline
\end{tabular}

\footnotetext{
- Neurons receiving a strongly orthodromic input along fimbria-fornix fibers (SOA cells) and neurons receiving a weak excitatory input along this pathway (WOA cells). Numbers in parentheses indicate the total number of cells tested. The values were obtained using electrodes which sampled nearly equal numbers of SOA and WOA cells.

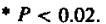



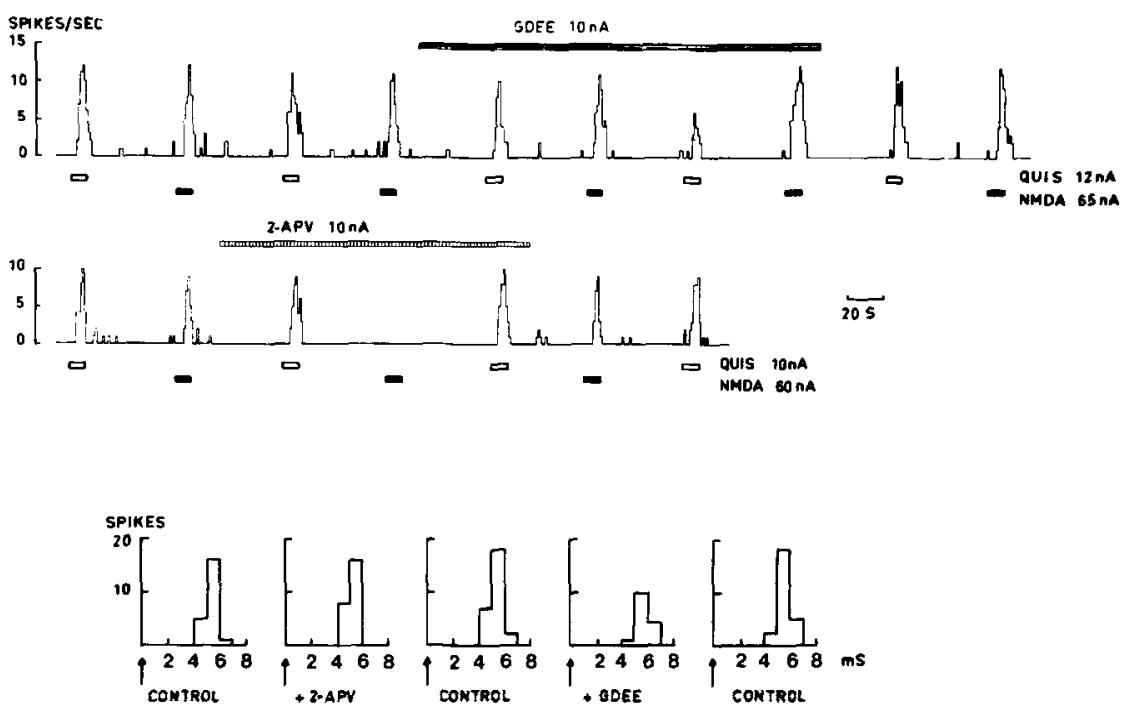

FIG. 2. The effect of glutamic acid diethylester (GDEE, upper tracing) and 2-amino-5-phosphonovaleric acid (2-APV, middle) on responses to exogenously applied $N$-methyl-D-aspartate (NMDA) and quisqualate (QUIS). Ordinates represent the number of spikes/s. Horizontal bars represent the duration of the applications. The computed poststimulus histograms (lower tracing) show the total number of responses of this neuron to $32 \mathrm{f}-\mathrm{fx}$ stimuli delivered before the release of the antagonist, during application of 2-APV (10 nA, $1 \mathrm{~min})$, during the control $5 \mathrm{~min}$ after termination of 2-APV release, during application of GDEE (10 $\mathrm{nA}, 1 \mathrm{~min})$, and during the control taken $5 \mathrm{~min}$ after GDEE administration. Arrows indicate the moment of stimulation.

excitations were temporarily suppressed by GDEE administered with currents between 10 and $50 \mathrm{nA}$ (see Table 2). In 8 of those neurons, the effect of GDEE on NMDA-induced responses was tested in addition. GDEE expelled with currents which antagonized responses to QUIS had no effect on NMDAevoked excitations in seven of the eight neurons. One neuron exhibited a slight but noticeable decrease in the NMDA response during iontophoretic application of GDEE.

Administration of 2-APV ( 5 to $20 \mathrm{nA}$ ) reversibly suppressed NMDAevoked excitations in all 18 cells tested. Selectivity of the antagonistic action by 2 -APV was tested in 11 cells. In 10 of those cells, 2-APV ejected with currents that diminished or completely abolished the NMDA responses, failed to affect QUIS-induced excitations (see an example in Fig. 2).

Effect of Glutamic Acid Diethylester and 2-Amino-5-phosphonovaleric Acid on the Action of $L$-Glutamate and L-Aspartate. Iontophoretically administered GDEE temporarily depressed GLU-induced responses in about $70 \%$ of the LSC neurons tested, whereas ASP-induced responses were diminished during release of the antagonists in about $50 \%$ of the cells (see Table 2). To obtain 
TABLE 2

The Effect of 2-APV and GDEE on Excitatory Responses Induced in Lateral Septal Neurons by NMDA, QUIS, ASP, and GLU ${ }^{a}$

\begin{tabular}{lcr}
\hline & GDEE & 2-APV \\
\hline NMDA & $1(8)$ & $18(18)$ \\
QUIS & $15(17)$ & $1(11)$ \\
ASP & $7(12)$ & $15(20)$ \\
GLU & $10(14)$ & $8(16)$ \\
\hline
\end{tabular}

a The table shows the number of neurons in which the antagonist listed at the head of the column reversibly reduced by at least $50 \%$ the response to the compound listed at the left. The numbers in parentheses show the total number of neurons in which the antagonistic action of 2-APV or GDEE on responses to the excitatory amino acids was tested.

antagonism of the natural amino acid responses, GDEE often had to be expelled with currents higher than the currents needed to demonstrate the blockade of the QUIS-induced responses. If the action of GDEE was tested on responses to GLU and ASP in the same neuron, GDEE blocked the responses to both amino acids equally well in six neurons. In two cells, the response to GLU was antagonized by GDEE with lower currents than the response to ASP, whereas the opposite effect was observed in two other cells. The 2-APV blocked the responses to ASP in about $75 \%$ of the neurons tested, whereas GLU-evoked excitations were antagonized by 2-APV in about $50 \%$ of the cells (see Table 2). The action of 2-APV on both GLU- and ASPevoked responses was tested in 11 neurons. In 6 of those neurons, the antagonist expelled with currents that effectively depressed ASP excitations had little or no effect on GLU-induced excitations. In the remaining 5 cells, the responses to ASP and GLU were antagonized by 2-APV with approximately the same currents.

Effect of Glutamic Acid Diethylester and 2-Amino-5-Phosphonovaleric Acid on Synaptically Evoked Excitation. The effect of GDEE on synaptically elicited excitations was studied in 18 SOA cells. In 15 of those cells, the release of the antagonist with currents which suppressed the QUIS- but not the NMDAinduced excitations, reversibly decreased the number of action potentials in response to fi-fx stimuli. Recovery of the number of synaptic responses in SOA cells after termination of GDEE administration was about $90 \%$. In 3 SOA cells, the synaptically induced activity was not affected by the release of GDEE.

Blockade of the fi-fx responses with 2-APV was attempted in nine SOA neurons. The 2-APV, ejected with currents which blocked responses to NMDA but not to QUIS, did not reduce the number of synaptically induced action 
potentials in any of those neurons. The antagonist consistently failed to affect fi-fx responses even when the expelling current was increased to $150 \mathrm{nA}$ for a long application period (about $7 \mathrm{~min}$ ). The selective blockade by GDEE of the synaptic excitations induced in SOA neurons by fi-fx stimulation was best demonstrated in cells in which the action of GDEE and 2-APV was examined on QUIS-, NMDA-, and synaptically induced responses (see Fig. 2).

\section{DISCUSSION}

As indicated by the rate of extracellularly recorded action potentials, iontophoretically administered GLU and ASP excited the majority of the LSC neurons tested. The GLU- and ASP-evoked excitations exhibited a similar rapid onset and fast decay as was reported for the effect of these substances in other brain regions $(4,17)$. Septal cells in which the spontaneous activity was temporarily depressed during release of $\mathrm{Mg}^{2+}$ ions, were still readily activated by GLU and ASP, although in the presence of $\mathrm{Mg}^{2+}$ ions the amino acid-induced responses tended to be slightly diminished. The latter aspect may be caused by the decrease in spontaneous activity during release of $\mathbf{M g}^{2+}$ ions, presumably by presynaptic suppression of transmitter release (24), but could also be due partly to the antagonistic postsynaptic action of $\mathrm{Mg}^{2+}$ ions on amino acid receptors, in particular the NMDA receptors (7). The above mentioned features of the extracellularly recorded responses to GLU and ASP would be expected if the amino acids acted on LSC cells as transmitters and activated some receptor-coupled ionic conductances $(1,12,35,36)$.

Considerably lower expelling currents were needed for GLU and ASP to excite SOA than WOA septal cells. Previous experiments (16) already showed that the inducement of excitations in SOA cells with GLU required much lower currents than in WOA cells. The weaker action of the amino acids on WOA cells could not be due to variations in the physical properties of the micropipets, to clogging of the micropipets, or to cell injury as a result of the penetrations. It was observed more than once that during one penetration SOA cells encountered in the deeper layers of the LSC were excited by the amino acids with currents in the same range as those needed to excite the more dorsally situated cells. The dorsal WOA cells on the other hand, required as high ejecting currents as did the more ventrally located WOA cells. Furthermore, LSC neurons show similarities in size and shape of the cell bodies, organization of the dendrites, and the sites of termination of the $\mathrm{f}-\mathrm{fx}$ fibers (26). Thus, the difference in sensitivity of SOA and WOA cells to GLU and ASP presumably reflects a difference in the distribution of amino acid receptors on the two groups of cells. This may be expected if the $\mathrm{fi}-\mathrm{fx}$ afferent fibers to the LSC utilize an amino acid as neurotransmitter. 
As indicated by the mean expelling currents and the mean GLU:ASP current ratio, GLU is a markedly stronger agent than ASP in SOA septal cells. Experiments by Gent and collaborators (14) showed that the number of micropipets expelling a greater amount of GLU than ASP at a given current was nearly equal to the number of electrodes that expelled more ASP than GLU. Because the SOA cells tested by us were collected with a rather high number $(N=13)$ of micropipet assemblies, it seems unlikely that a consistently higher release of GLU than of ASP could account entirely for the observed stronger excitatory action of GLU.

In summary, these results show that the amino acid receptors on LSC cells and on SOA cells in particular, are more readily activated by GLU than by ASP.

The majority of the LSC cells were excited by QUIS and NMDA; GDEE suppressed the QUIS- but not the NMDA-induced responses, whereas 2APV reversibly abolished the responses to NMDA but not to QUIS [see also $(6,21)]$. The selective blockade of the QUIS and NMDA responses with the appropriate antagonists indicates that in the LSC, QUIS, and NMDA act on different types of amino acid receptors. Neurons of the LSC carry both the QUIS and NMDA types of receptors. For a small number of neurons tested, QUIS-expelling currents were significantly lower in SOA cells than in WOA cells. This suggests that the distribution of QUIS receptors on LSC cells seems to be at least related to the intensity of the excitatory input.

The GDEE and 2-APV, ejected with currents that abolished responses to QUIS and NMDA, respectively, frequently failed to depress GLU- and ASPevoked excitations completely. This may be explained at least partly by the fact that iontophoretically administered GLU and ASP behave as mixed agonists for the QUIS, NMDA, and KA types of receptors [see review (5)]. Thus, blockade of the QUIS receptor with GDEE will presumably decrease the number of QUIS receptors involved in the excitations elicited by GLU and ASP. However, due to the mixed agonism of these amino acids, GLU and ASP may still induce excitation by acting on NMDA receptors. The mixed agonistic action of GLU and ASP on QUIS and NMDA receptors makes it difficult to establish from iontophoretic experiments such as ours which of the two amino acids is the natural ligand for the QUIS receptor and which is that for the NMDA receptor. Solving this problem is essential if the neurotransmitter acting at a given pathway is to be identified by means of antagonists. In our experiments on LSC cells, 2-APV frequently antagonized ASP-induced responses better than GLU-evoked excitations, whereas GDEE more often blocked the responses to GLU more effectively than those to ASP. This observation, which is in agreement with other studies $(6,22)$ supports the idea (5) that GLU is presumably the endogenous ligand for the QUIS receptor, and ASP may be the natural agonist for the NMDA receptor. 
The GDEE, expelled with currents that suppressed the responses to exogenously applied QUIS, reversibly diminished or suppressed the excitations elicited in SOA cells by fi-fx stimuli. On the other hand, 2-APV consistently failed to reduce the number of action potentials induced in SOA neurons by stimulation of the fi-fx, even when the antagonist was expelled with high currents for a long period of time. It is known from the literature $(8,15,22)$ that GDEE does not block the KA receptors. Our results therefore indicate that the QUIS receptor and not the NMDA or KA receptors mediate transmission between fi-fx fibers and SOA septal cells. Because GLU-induced excitations in the LSC seem to be mainly mediated through QUIS receptors, it may be inferred from the latter observations that GLU and not ASP acts as a neurotransmitter in this projection.

Our study shows that LSC neurons, in particular those neurons receiving strongly orthodromic input along fi-fx fibers, are more readily activated by GLU than by ASP. Furthermore, it appears that QUIS receptors are involved in monosynaptic excitations elicited in LSC neurons by $\mathrm{fi}-\mathrm{fx}$ stimuli and that GLU-induced excitations in the LSC are presumably mediated by these QUIS receptors.

These results support biochemical data $(18,32)$ and provide additional electrophysiologic evidence (16) that neurotransmission between fi-fx fibers and LSC neurons is mediated by an excitatory amino acid. Moreover, they suggest that GLU rather than ASP acts as transmitter in this projection. This is in agreement with histochemical, electrophysiologic, and pharmacologic evidence indicating that GLU is the transmitter in the commissural and associational fibers of the CA3 pyramidal cells in the hippocampus (27-29). Because multiple fluorescent labeling showed that the majority of the hippocampal fibers projecting to the LSC originate in the very same population of CA3 cells (31), the same transmitter may be expected to be active in these projections. Yet, evidence based on intracellular experiments and showing that GLU or a related amino acid can act as neurotransmitter on LSC cells innervated by $\mathrm{fi}-\mathrm{fx}$ fibers remains to be provided.

\section{REFERENCES}

1. Altmann, H., G. ten Bruggencate, P. Pickelmann, and R. Steinberg. 1976. Effects of glutamate, aspartate, and two presumed antagonists on feline rubrospinal neurones. Eur. J. Physiol. 364: 249-255.

2. BALCAR, V. J., AND G. A. R. JOHNSTON. 1972. The structural specificity of the high affinity uptake of L-glutamate and L-aspartate by brain slices. J. Neurochem. 24: 2657-2666.

3. Biscoe, T. J., P. M. HeAdly, D. Lodge, M. R. MARTin, AND J. C. Watkins. 1976. The sensitivity of rat spinal interneurones and Renshaw cells to L-glutamate and L-aspartate. Exp. Brain Res. 26: 547-551.

4. Curtis, D. R., A. W. Duggan, D. Felix, G. A. R. Johnston, A. K. Tebēcis, and J. C. 
WATKINS. 1972. Excitation of mammalian central neurones by acidic amino acids. Brain Res. 41: 283-301.

5. Davies, J., R. H. Evans, A. A. Francis, A. W. JoneS, AND J. C. Watkins. 1980. Excitatory amino acid receptors in the vertebrate central nervous system. Pages 333-347 in U. Z. LitTAuER, Y. DudaI, I. Silman, V. I. TeichBERG, AND Z. Vogel, Eds., Neurotransmitters and Their Receptors. Wiley, New York.

6. Davies, J., A. A. Francis, A. W. Jones, and J. C. Watkins. 1981. 2-amino-5-phosphonovalerate (2APV), a potent and selective antagonist of amino acid induced and synaptic excitation. Neurosci. Lett. 21: 77-81.

7. DAVIES, J., AND J. C. WATKINS. 1977. Effect of magnesium ions on the responses of spinal neurones to excitatory amino acids and acetylcholine. Brain Res. 130: 364-368.

8. DAvieS, J., AND J. C. WATKINS. 1979. Selective antagonism of amino acid-induced and synaptic excitation in the cat spinal cord. J. Physiol. (London) 297: 621-635.

9. DeFrancE, J. F., S. T. KITAI, AND T. ShimONO. 1973. Electrophysiological analysis of the hippocampal-septal projections. I. Response and topographical characteristics. Exp. Brain Res. 17: 447-462.

10. DeFrance, J. F., S. T. Kital, ANd T. Shimono. 1973. Electrophysiological analysis of the hippocampal-scptal projections. II. Functional charactcristics. Exp. Brain Res. 17: 463476.

11. DugGan, A. W. 1974. The differential sensitivity to L-glutamate and L-aspartate of spinal interneurones and Renshaw cells. Exp. Brain Res. 19: 522-528.

12. Engberg, I., J. A. Flatman, AND J. D. C. LamberT, 1978. The action of $N$-methyl-Daspartic and kainic acids on motoneurones with emphasis on conductance changes. Proceedings, British Pharmacological Society, September 13-15, 1978, 385 P.

13. FonNUM, F., and I. WALAAS. 1978. The effect of intrahippocampal kainic acid injections and surgical lesions on neurotransmitters in hippocampus and septum. $J$. Neurochem. 31: $1173-1181$.

14. Gent, J. P., R. Morgan, AND J. H. WolstenCROFT. 1974. Determination of relative potency of two excitant amino acids. Neuropharmacology 13: 441-447.

15. Hicks, T. P., J. G. HALL, AND H. MCLENNAN. 1978. Ranking of excitatory amino acids by the antagonists glutamic acid diethylester and D- $\alpha$-amino adipic acid. Can. J. Physiol. Pharmacol. 56: 901-906.

16. JOËLS, M., AND I. J. A. URBAN. 1984. Electrophysiological and pharmacological evidence in favor of amino acid neurotransmission in fimbria-fornix fibers innervating the lateral septal complex of the rat. Exp. Brain Res., in press.

17. Johnson, J. L., 1972. Glutamic acid as a synaptic transmitter in the nervous system. A review. Brain Res. 37: 1-19.

18. Malthe-SøRENSSEN, D., K. K. SKREDE, AND F. FonNUM. 1980. Release of D- ${ }^{3} H$-aspartate from the dorsolateral septum after electrical stimulation of the fimbria in vitro. Neuroscience 5: $127-133$.

19. MCCulloch, R. M., G. A. R. Johnston, C. J. A. GAme, and D. R. CuRTis. 1974. The differential sensitivity of spinal interneurones and Renshaw cells to kainate and $\mathrm{N}$-methylD-aspartate. Exp. Brain Res. 21: 515-518.

20. MCLENNAN, H. 1981. On the nature of the receptors for various excitatory amino acids in the mammalian central nervous system. Pages 253-262 in G. L. GESSA AND G. DICHIARA, Eds., Glutamate as a Neurotransmitter, Advances in Biochemical Psychopharmacology, Vol. 27. Raven Press, New York.

21. MCLENNAN, H., AND L. LIU. 1982. The action of six antagonists of the excitatory amino acids on neurones of the rat spinal cord. Exp. Brain Res. 45: 151-156. 
22. MCLENNAN, H., AND D. LODGE. 1979. The antagonism of amino acid-induced excitation of spinal neurones in the cat. Brain Res. 169: 83-90.

23. MCLenNAN, H., AND J. J. MiLleR. 1974. The hippocampal control of neuronal discharges in the septum of the rat. J. Physiol (London) 237: 607-624.

24. NiCOLL, R. A. 1971. Pharmacological evidence for GABA as the transmitter in the granule cell inhibition in the olfactory bulb. Brain Res. 35: 137-149.

25. NITSCI, C., Y. KIM, C. SHIMADA, AND Y. OKaDA. 1979. Effect of hippocampus extirpation in the rat on glutamate levels in target structures of hippocampal efferents. Neurosci. Lett. 11: 295-299.

26. RaisMAN, G. 1969. A comparison of the mode of termination of the hippocampal and hypothalamic afferents to the septal nuclei as revealed by electron microscopy of degeneration Exp. Brain Res. 7: 317-343.

27. SCHWARTZKROIN, P. A., AND P. ANDERSEN. 1975. Glutamic acid sensitivity of dendrites in hippocampal slices in vitro. Adv. Neurol. 12: 45-51.

28. SEGAL, M. 1976. Glutamate antagonists in rat hippocampus. Br. J. Pharmacol. 58: 341345.

29. STORM-MATHISEN, J., AND L. L. IVERSEN. 1979. Uptake of ${ }^{3} \mathrm{H}$-glutamic acid in excitatory nerve endings: light and electron microscopic observations in the hippocampal formation of the rat. Neuroscience 4: 1237-1253.

30. Storm-MATHISEN, J., AND M. WOXEN OPSAHL. 1978. Aspartate and/or glutamate may be transmitters in hippocampal efferents to septum and hypothalamus. Neurosci. Lett. 9: 65-70.

31. Swanson, L. W., P. E. Sawchenko, And W. M. Cowan. 1980. Evidence that the commissural, associational and septal projections of the regio inferior of the hippocampus arise from the same ncurons. Brain Res. 197: 207-212.

32. WALAAS, I., AND F. FONNUM. 1980. Biochemical evidence for glutamate as a transmitter in hippocampal efferents to the basal forebrain and hypothalamus in the rat brain. NeUroscience 5: 1291-1698.

33. Watkins, J. C., AND R. H. Evans. 1981. Excitatory amino acid transmitters. Annu. Rev. Pharmacol. Toxicol. 21: 165-204.

34. ZACZEK, R., J. C. HEDREEN, AND J. T. COYLE. 1979. Evidence for a hippocampal septal glutamatergic pathway in the rat. Exp. Neurnl. 65: 145-156.

35. ZieglgänSBerger, W., AND J. Champagnat. 1979. Cat spinal motoneurones exhibit topographic sensitivity to glutamate and glycine. Brain Res. 160: 95-104.

36. ZIEGLGÄNSBERGER, W., AND E. A. PUIL. 1973. Actions of glutamic acid on spinal neurons. Exp. Brain Res. 17: 35-49. 